\title{
Gut Microbiota in Health and Disease - An Overview
}

\author{
Shrihari TG*, Trishna Saikia, Vijeev Vasudevan, Devaraju D and Himanshi Tomar \\ Department of Oral medicine and Radiology, India \\ *Corresponding author: Shrihari TG, Department of Oral medicine and Radiology, Bengaluru, Karnataka, India
}

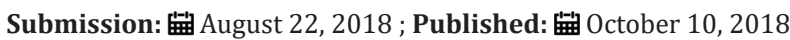

\begin{abstract}
Gut microbiota refers to the population of microorganisms that inhabit the mucous membrane of the intestines of normal healthy persons. The gut microbiota is of vital importance for health as it helps in maintaining the homeostasis. Still, some ecological changes in the microbiota enable pathogens to grow explicitly and cause disease. A grasping of the environment of gut and interactions amongst microbiota guide towards the foremost cause of start of diseases. Our future holistic health depends on maintaining normal gut microbiota rather than treating with reductionist chemical drugs which have many adverse effects and cumbersome. This article briefs about the role of gut microbiota in health and disease.
\end{abstract}

Keywords: SCF's (Short chain fatty acids); Autoimmune disease; Cancer; Dysbiosis; NF-kB; Infectious diseases; Microbiota- gut- brain axis; Oral microbiota

Abbreviations: IL-1, IL-2, IL-6, IL-12: Interleukin-1, Interleukin-2, Interleukin-6, Interleukin-12; TNF-a: Tumor Necrosis Factor- Alpha; NF-kB: Nuclear Factor- Kappa B Receptor; STAT-3: Signal Transducer and Activator of Transcription-3; TLR: Toll Like Receptor; Th1, Th2: Type 1 Helper Cells, Type 2 Helper Cells; MMP -2,9: Matrix-Metallo Proteases; CDI: C Difficile Infection; BT: Bacterial Translocation; MDD: Major Depressive Disorder

\section{Introduction}

In humans there are 100 trillion germs, whereas only 23,000 genes [1]. Our health mainly depends on gut symbiotic microorganisms known as germinomes such as viruses, bacteria, protozoa, parasites, fungi and are called human metagenome [2]. These maintain homeostatic environment of our body by maintaining mucosal integrity, acts against pathogens by producing antimicrobial substance like lactic acid, improves immunity by yielding cytokines like IL-2, IL-12. Anti-inflammatory role by the production of short-chain fatty acids such as butyrate, acetate, propionate from dietary fibers mainly present in vegetables and fruits involved in the preventive and therapeutic role in large bowel disorders, cardiovascular disorders, reduction of cholesterol, type 2 diabetes, obesity $[2,3]$.

Dysbiosis is a state of altered microbiota involved in various diseases such as psychological disorders, cancer, skin disorders, obesity, autoimmune diseases, cardiovascular disorders, diabetes, gastrointestinal disorders, and infectious diseases such as bacterial, viral and fungal lesions by altering the normal homeostatic environment of the human body [3]. Dysbiosis results in altered immune status by increasing inflammatory cytokines such as TNF-œ, IL-1, mediated activation of a key transcription factor $\mathrm{NF}-\mathrm{kB}$, involved in chronic inflammation, cancer, altered immune response (immune modulation) [4].

Stress mediated release of neuropeptides such as corticotropic releasing hormone and noradrenaline through HPA -axis mediated dysbiosis activates inflammatory mediators such as IL$1 ß$, TNF- $œ, 1 \mathrm{~L}-6$, pro-inflammatory cytokines further activates key transcription factors NF-kB, STAT-3, through recognition of microbes and microbial products by TLR(Toll like Receptor) expressed on macrophages and dendritic cells of innate immune cells involved in chronic inflammation, immune modulation by altered $\mathrm{T}$ regs, which is involved in self-tolerance and immune homeostasis, conversion of Th1 to Th2 type lymphocytes, release of proteolytic enzymes such as matrix-metallo proteases(mmp-2,9) leads to tissue damage and cellular changes results in autoimmune disease [5]. Gut microbiota is involved in the development of cellular mucosal immunity and humoral immunity. The metabolites and signals of microorganisms sensed by the hematopoietic and nonhematopoietic cells of the innate immune system and translated into physiological responses. Smoking and lack of physical activity result in a change in microflora such as bacteroids, prevotella of large bowel increase the risk factor for colorectal cancer [6].

\section{Discussion}

\section{The human microbiota in health}

The host physiology is affected by the human microbiota to a considerable amount. The human body is colonized by trillions of microbes such as bacteria, archaea, viruses etc, consisting at least 1000 different species [7]. Shortly after birth, the human body is colonized by the commensal bacteria, which gradually develops into a diverse ecosystem during the growth period of the host. 
With time, the association of the host and bacteria develop into an advantageous relationship [7,8]. Symbiotic microorganisms help in the metabolization of indigestible compounds, provide critical nutrients and help in defending against settlement by opportunistic organisms thereby forming the framework of the intestinal layout [8].

Maintaining a normal state of human health mainly depends on the microbiota of human body especially gut microbiota, which is a military system of human body. Dietary habits such as dietary fibers, proteins, carbohydrates, psychological stress, smoking, obesity, physical exercise, influence the gut microbiota [9]. Gut microbiota consists of bacteroids, actinobacteria, yeast, single-cell eukaryotes, viruses and parasitic worms. Bifidobacterias, lactobacillus, produce vitamins such as vitamin-k, vitamin b12, biotin, folate, thiamine, and minerals such as calcium, magnesium, phosphate [9]. The colonic microbiota consists of bacteroids, prevotella, rhuminococcus [10]. Additionally, gut-colonizing bacteria help in the normal development of humoral and cellular mucosal immune systems. However, under certain conditions, some microbiota can induce inflammation [10].

A microbiome can only be kept in health via proper oral hygiene and with a sound immune system [11]. The oral cavity has over 700 types of bacteria which bestow to the health and physiologic position of the oral cavity [11]. The bacterial colonies in heath and diseased state of the oral cavity are quite different, which suggests that there might be a basic oral microbiota of health [12]. The cue to the health of oral cavity is a well-balanced and manifold microbiota that executes commensalism amongst themselves and mutualism with its host [12]. Moreover, the correlation between the microbiota and its host in health is valuable, as the host is furnishing its microbial colonies with a habitat in which they can flourish and thereby help in keeping their host in good health [13]. The microorganisms in health stop progression of disease in many ways, like preventing the attachment of pathogens in particular places by holding a slot liked by a pathogen, which prevents a pathogen from inhabiting a preoccupied place, which hampers a pathogen's capacity to flourish, and corrupt their pathogenicity $[13,14]$.

\section{The human microbiota in disease}

Dysbiosis is a state of alteration in normal symbiotic microorganisms due to various factors which results in various diseases [15]. One of the most common diseases caused by dysbiosis is an infection [15]. Treatment of infectious diseases has an impact on the human microbiota which determines the outcome of the infectious disease in the human host [16]. The intestinal mucosa is colonized by the offending organisms which result in the formation of a strong inflammatory response, followed by the translocation of the intestinal bacteria [17]. Clostridium difficile is an anaerobic, gram-positive, spore-producing bacillus which is a component of the human gut microbiota [18]. The most common complication followed by administration of antibiotics is the pathological overgrowth of Clostridium difficile and is related to antibiotic- associated diarrhea [18]. The homeostasis of the intestinal mucosa is disturbed hence decreasing the resistance against toxinfabricating C. difficile and encouraging the development of CDI (C difficile Infection) [19].

There is a close interaction between liver and gut microbes, as the gut microbes generate ethanol, ammonia, and acetaldehyde which affect liver function through endotoxin release or liver metabolism [20]. Any changes in the gut microbiota play an essential role in the induction, promotion, and progression of liver disease [20]. The gut microbiota is engaged in the pathologic process of liver cirrhosis complications, such as infections, spontaneous bacterial peritonitis, hepatic encephalopathy, and renal failure [19,20]. Liver disease is associated with an increase in Enterobacteriaceae and a decrease in Bifidobacterium. Dysbiosis of the gut can lead to endotoxemia in patients via bacterial translocation (BT) [21]. Endotoxemia may encourage immune dysfunction, hence leading to further liver cell necrosis and liver failure $[21,22]$. Therefore, it's necessary for the development of new probiotics specifically for the prevention, progression, and treatment of liver diseases [23].

Helicobacter pylori-related chronic inflammation is believed to be the potential risk factor for gastric carcinoma [24]. Approximately, each year 660000 new cases of gastric carcinoma are provoked by Helicobacter pylori contamination, resulting in dropping of acidfabricating parietal cells, hence leading to abdominal degeneration, metaplasia, dysplasia, and, finally, the formation of cancer [24]. The carcinogenic potential may be associated with the genetic diversity of the H. pylori strain, changes in host responses, and specific hostmicrobe interactions [25]. The phylogenetic origin of Helicobacter pylori is a good indicator of the risk for gastric carcinoma. The interaction of the gut microbiota with the formation of colon cancer has recently become a major focus of research. Microbial dysbiosis has been related to the etiology of colorectal adenomas and carcinomas. Studies which were done in the recent times suggests that chronic inflammation at the end of the esophagus, caused by gastroesophageal reflux is associated with esophageal adenocarcinoma (EA). The pathophysiology of this process can be described as gastroesophageal reflux disease-Barrett's esophagusesophageal adenocarcinoma (GERD-BE-EA) [26,27].

Obesity and its associated metabolic complications, such as type 2-diabetes and cardiovascular disease, have become a global epidemic health problem and are the consequences of a complex multidirectional interaction among host genetics, diet, environment, and the gut microbiota $[28,29]$. Growing numbers of studies indicate that an alteration of gut microbiota designated by lesser diversity and flexibility is related to diabetes. The mechanisms that cause the disease may be related to the translocation of microbiota from the gut to the tissues, thus inducing inflammation [30]. Obesity and its associated metabolic complications may be a result of complex gene-environment interactions. Microbiome interventions aimed at restoring the homeostasis of the gut microbiome have recently emerged, such as the ingestion of specific fibers or therapeutic microbes $[30,31]$. These are promising strategies to reduce insulin resistance and related metabolic diseases [32]. 
The initiating factors causing a biological switch are poor oral hygiene, weakened immune system, and genetics. Negligence in the removal of plaque build-up will cause profusion of bacteria which may turn pathogenic, lessen multifariousness of the oral cavity, resulting in diseases like dental caries and periodontal disease [33,34]. Anaerobic microorganisms found in the tongue crypts can also overgrow and cause halitosis, or bad breath $[35,36]$. Maintaining absolute oral hygiene is important because it helps in preventing oral disease. Oral diseases like dental cavity and periodontitis are amongst the widespread diseases worldwide affecting people of all age groups and geographic populations $[37,38]$. Periodontal disease can arise from the accumulation of subgingival plaque resulting in a change of the oral microorganisms from a healthy condition to a diseased condition $[39,40]$. Numerous studies conducted worldwide exhibited bad oral hygiene and tooth loss leading to rising in abdominal, pancreatic, and other types of cancers [41].

Psychiatric diseases are a major threat to mankind, caused by biological, environmental, psychological factors. The gut-brain axis helps in maintaining normal gastrointestinal and brain function. Gut microbiota plays a very important role in maintaining microbiotagut-brain axis, involving endocrine system, neural system, immune system, metabolic system and their interaction with each other [42]. Dysbiosis of gut microbiota may result in translocation of the microbiota of gut to the mesenteric lymphoid tissue via the intestinal wall, initiate an immune response that can lead to a release of pro-inflammatory cytokines and neuronal activation of spinal afferent neurons and vagus nerve. Autism spectrum disorders and MDD (Major Depressive Disorder) are associated with gut microbial dysbiosis. Further research is on interaction between host, gut-microbiota, hormonal, genetic, genetic changes in the development of autism and MDD [1,42].

\section{Conclusion}

The gutmicrobiota consists of various symbioticmicroorganisms which help in maintaining a normal homeostatic environment of the human body. The next important stage is to explore the functions of the microbiota further in detail. This will help in understanding the host-microbiota interrelationship in health and disease, and lead to treatment which focuses on the microbiota to maintain health and to treat a variety of diseases. Understanding of gut microbiota, environment, an interaction between host and microorganisms and their role in health and disease helps in future therapeutic purpose.

\section{References}

1. Baohong W, Mingfei Y, Longxian L, Zongxin L, Lanjuan L, et al. (2017) The human microbiota in health and disease. Engineering 3(1): 71-82.

2. Franzosa EA, Morgan XC, Segata N, Waldron L, Reyes J, et al. (2014) Relating the metatranscriptome and metagenome of the human gut. Proc Natl Acad Sci USA 111(22): E2329-E2338.

3. Yamashiro Y (2017) Gut microbiota in health and disease. Ann Nutr Metab 71(3-4): 242-246.

4. Icaza-Chávez ME (2013) Gut microbiota in health and disease. Revista de Gastroenterología de México 78(4): 240-248.
5. Tremaroli V, Bäckhed F (2012) Functional interactions between the gut microbiota and host metabolism. Nature 489(7415): 242-249.

6. Castellarin M, Warren RL, Freeman JD, Dreolini L, Krzywinski M, et al. (2012) Fusobacterium nucleatum infection is prevalent in human colorectal carcinoma. Genome Res 22(2): 299-306.

7. Burcelin R (2016) Gut microbiota and immune crosstalk in metabolic disease. Mol Metab 5(9): 771-781.

8. Ursell LK, Haiser HJ, Van Treuren W, Garg N, Reddivari L, et al. (2014) The intestinal metabolome: an intersection between microbiota and host. Gastroenterology 146(6): 1470-1476.

9. Round JL, Mazmanian SK (2009) The gut microbiota shapes intestinal immune responses during health and disease. Nat Rev Immunol 9(5): 313-323.

10. Grehan MJ, Borody TJ, Leis SM, Campbell J, Mitchell H, et al (2010) Durable alteration of the colonic microbiota by the administration of donor fecal flora. J Clin Gastroenterol 44(8): 551-561.

11. Zarco MF, Vess TJ, Ginsburg GS (2012) The oral microbiome in health and disease and the potential impact on personalized dental medicine. Oral Dis 18(2): 109-120.

12. Rodrigues Hoffmann A, Proctor LM, Surette MG, Suchodolski JS (2016) The microbiome: The trillions of microorganisms that maintain health and cause disease in humans and companion animals. Vet Pathol 53(1): $10-21$

13. Integrative HMP (iHMP) Research Network Consortium (2014) The integrative human microbiome project: Dynamic analysis of microbiome-host omics profiles during periods of human health and disease. Cell Host Microbe 16(3): 276-289.

14. Yost S, Duran Pinedo AE, Teles R, Krishnan K, Frias Lopez J (2015) Functional signatures of oral dysbiosis during periodontitis progression revealed by microbial metatranscriptome analysis. Genome Med 7(1): 27.

15. Xiong W, Giannone RJ, Morowitz MJ, Banfield JF, Hettich RL (2015) Development of an enhanced metaproteomic approach for deepening the microbiome characterization of the human infant gut. J Proteome Res 14(1): 133-141.

16. Faith JJ, Rey FE, O’Donnell D, Karlsson M, McNulty NP, et al. (2010) Creating and characterizing communities of human gut microbes in gnotobiotic mice. ISME J 4(9): 1094-1098.

17. Human Microbiome Project C (2012) A framework for human microbiome research. Nature 486(7402): 215-221.

18. Khoruts A, Dicksved J, Jansson JK, Sadowsky MJ (2010) Changes in the composition of the human fecal microbiome after bacteriotherapy for recurrent Clostridium difficile-associated diarrhea. J Clin Gastroenterol 44(5): 354-360.

19. Silverman MS, Davis I, Pillai DR (2010) Success of self-administered home fecal transplantation for chronic Clostridium difficile infection. Clin Gastroenterol Hepatol 8(5): 471-473.

20. De Martel C, Ferlay J, Franceschi S, Vignat J, Bray F, et al. (2012) Global burden of cancers attributable to infections in 2008: a review and synthetic analysis. Lancet Oncol 13(6): 607-615.

21. Voigt RM, Forsyth CB, Green SJ, Mutlu E, Engen P, et al. (2014) Circadian disorganization alters intestinal microbiota. PLoS One 9(5): e97500.

22. Frazier TH, DiBaise JK, McClain CJ (2011) Gut microbiota, intestinal permeability, obesity induced inflammation, and liver injury. JPEN J Parenter Enteral Nutr 35(5 Suppl): 14S-20S.

23. Nardone G, Rocco A (2004) Probiotics: A potential target for the prevention and treatment of steatohepatitis. J Clin Gastroenterol 38(6): 121-122. 
24. De Sablet T, Piazuelo MB, Shaffer CL, Schneider BG, Asim M, et al. (2011) Phylogeographic origin of Helicobacter pylori is a determinant of gastric cancer risk. Gut 60(9): 1189-1195. (2008) Relationship between Helicobacter pylori infection and gastric atrophy and the stages of the oesophageal inflammation, metaplasia adenocarcinoma sequence: Results from the FINBAR case-control study. Gut 57(6): 734-739.

26. Franks PW, McCarthy MI (2016) Exposing the exposures responsible for type 2 diabetes and obesity. Science 354(6308): 69-73.

27. Cani PD, Osto M, Geurts L, Everard A (2012) Involvement of gut microbiota in the development of low-grade inflammation and type 2 diabetes associated with obesity. Gut Microbes 3(4): 279-288.

28. Sanapareddy N, Legge RM, Jovov B, McCoy A, Burcal L, et al. (2012) Increased rectal microbial richness is associated with the presence of colorectal adenomas in humans. ISME J 6(10): 1858-1868.

29. Clavel T, Desmarchelier C, Haller D, Gérard P, Rohn S, et al. (2014) Intestinal microbiota in metabolic diseases: from bacterial community structure and functions to species of pathophysiological relevance. Gut Microbes 5(4): 544-551.

30. O'Hara AM, Shanahan F (2006) The gut flora as a forgotten organ. EMBO Rep 7(7): 688-693.

31. Brenchley JM, Douek DC (2012) Microbial translocation across the GI tract. Annu Rev Immunol 30: 149-173.

32. Anders HJ, Andersen K, Stecher B (2013) The intestinal microbiota, a leaky gut, and abnormal immunity in kidney disease. Kidney Int 83(6): 1010-1016.

33. Dethlefsen L, Relman DA (2011) Incomplete recovery and individualized responses of the human distal gut microbiota to repeated antibiotic perturbation. Proc Natl Acad Sci USA 108(1): 4554-4561.
25. Anderson LA, Murphy SJ, Johnston BT, Watson RG, Ferguson HR, et al.

34. Vijay Kumar M, Aitken JD, Carvalho FA, Cullender TC, Mwangi S, et al. (2010) Metabolic syndrome and altered gut microbiota in mice lacking Toll-like receptor 5. Science 328(5975): 228-231.

35. Martin FP, Sprenger N, Montoliu I, Rezzi S, Kochhar S, et al. (2010) Dietary modulation of gut functional ecology studied by fecal metabonomics. J Proteome Res 9(10): 5284-5295.

36. Ravel J, Gajer P, Abdo Z, Schneider GM, Koenig SS, et al. (2011) Vaginal microbiome of reproductive-age women. Proc Natl Acad Sci USA 108(1): 4680-4687.

37. Sun Y, Cai Y, Huse SM, Knight R, Farmerie WG, et al. (2012) A large-scale benchmark study of existing algorithms for taxonomy-independent microbial community analysis. Brief Bioinform 13(1): 107-121.

38. Castellarin M, Warren RL, Freeman JD, Dreolini L, Krzywinski M, et al. (2012) Fusobacterium nucleatum infection is prevalent in human colorectal carcinoma. Genome Res 22(2): 299-306.

39. Hu Z, Zhang Y, Li Z, Yu Y, Kang W, et al. (2016) Effect of Helicobacter pylori infection on chronic periodontitis by the change of microecology and inflammation. Oncotarget 7(41): 66700-66712.

40. Hand TW, Dos Santos LM, Bouladoux N, Molloy MJ, Pagán AJ, et al. (2012) Acute gastrointestinal infection induces long-lived microbiota-specific $\mathrm{T}$ cell responses. Science 337(6101): 1553-1556.

41. Jung Lee W, Lattimer LD, Stephen S, Borum ML, Doman DB (2015) Fecal microbiota transplantation: A review of emerging indications beyond relapsing clostridium difficile toxin colitis. Gastroenterol Hepatol (N Y) 11(1): 24-32.

42. Rogers GB, Keating DJ, Young RL, Wong ML, Licinio J, et al. (2016) From gut dysbiosis to altered brain function and mental illness: mechanisms and pathways. Mol Psychiatry 21(6): 738-748.

Creative Commons Attribution 4.0 International License

For possible submissions Click Here Submit Article

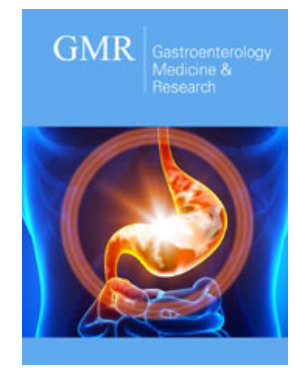

Gastroenterology Medicine \& Research

\section{Benefits of Publishing with us}

- High-level peer review and editorial services

- Freely accessible online immediately upon publication

- Authors retain the copyright to their work

- Licensing it under a Creative Commons license

- Visibility through different online platforms 